\title{
Convivencia escolar y solución de conflictos mediante la investigación como estrategia pedagógica

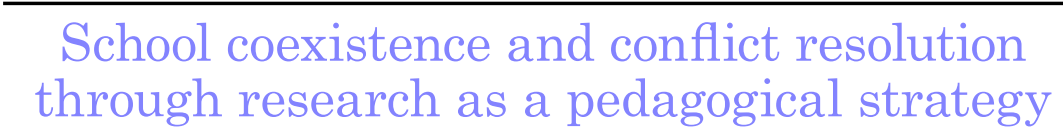

DOI: http://dx.doi.org/10.17981/cultedusoc.9.3.2018.0

Artículo de investigación. Fecha de recepción: 15/06/2018. Fecha de aceptación: 27/11/2018

\section{Sallid Contreras-Rodríguez; Neyide Colón-Luna; Catalina Gonzales - Montalvo; Plinio Machado-De la Cruz; María Melo-Vásquez, Luz Vergara-Luna ${ }^{1}$}

IED Tercera Mixta de la Sede Adriano (Colombia)

Para citar este artículo:

Contreras-Rodríguez, S., Colón-Luna, N., Gonzales-Montalvo, C., Machado-De la Cruz, P., Melo-Vásquez, M. y Vergara-Luna, M. (2018). Convivencia escolar y solución de conflictos mediante la investigación como estrategia pedagógica. Cultura. Educación y Sociedad 9(3), 63-72. DOI: http://dx.doi.org/10.17981/cultedusoc.9.3.2018.0

\section{Resumen}

La convivencia escolar permite la construcción de ambientes libres de violencia, enmarcados en la tolerancia y el respeto hacia los derechos del otro en la escuela, fomentando la participación activa de los estudiantes para generar estrategias de solución de conflictos mediante el uso de estrategias pedagógicas innovadoras que fomenten el interés en los jóvenes. El estudio tuvo como propósito fortalecer la convivencia escolar y solución de conflictos mediante la investigación como estrategia pedagógica. La metodología se orientó bajo el enfoque cualitativo, utilizando el tipo de investigación acción, desde un diseño descriptivo, exploratorio. Para la recolección de datos se utilizaron técnicas como observación participante y diario de campo. La población estuvo conformada por ciento ochenta (180) estudiantes de básica primaria con edades que oscilan entre los 5 y 15 años de la IED Tercera Mixta de la Sede Adriano Puentes del municipio de Fundación Magdalena. Los resultados evidenciaron el diseño de estrategias de formación en gestores de paz implementando la Investigación como estrategia pedagógica.

Palabras clave: Convivencia escolar, solución de conflictos, investigación como estrategia pedagógica.

\section{Abstract}

School coexistence allows the construction of violence-free environments, framed in tolerance and respect for the rights of the other in school, encouraging the active participation of students to generate strategies for conflict resolution through the use of innovative pedagogical strategies that Promote interest in young people. The purpose of the study was to strengthen school coexistence and conflict resolution through research as a pedagogical strategy. The methodology was oriented under the qualitative approach, using the type of action research, from a descriptive, exploratory design. For data collection techniques were used as participant observation and field diary. The population was made up of one hundred and eighty (180) elementary school students with ages ranging from 5 to 15 years of the Third Mixed IED Adriano Puentes Headquarters in the municipality of Magdalena Foundation. The results showed the design of training strategies in peace managers implementing Research as a pedagogical strategy.

Keywords: School coexistence, conflict resolution, research as a pedagogical strategy. 


\section{Introducción}

Para el hombre, desde que nace es fundamental la socialización entre los individuos como resultado de estas interacciones el sujeto se desarrolla a nivel cognitivo, cognoscitivo, psíquico y socialmente, además de hacerse imprescindible para la salud mental y la integridad física. A su vez aprende y aprehende actitudes del grupo primario y de los entornos en los que tiene participación. Desde la perspectiva holística que caracteriza al ser humano, se comprende que la conducta es el resultado del dinamismo, correlación e interactividad de los factores antes descritos, enmarcados en un contexto especifico, reflejándose en el desenvolvimiento de las relaciones interpersonales e intrapersonal y en las diversas esferas que lo conforma.

Valenzuela y Rochin ( 2013) aseguran que,

la libertad de expresión, contribuye a que existan otros derechos fundamentales como la libertad de prensa, los derechos de reunión, los derechos de asociación, de petición y de participación política (p. 94).

Es de destacar que, la convivencia escolar debe contar con pilares sustanciales como el respeto, la honestidad, la tolerancia y la solidaridad, asimismo normas y los códigos de comportamientos que orienten el conducir del grupo. En las instituciones educativas cada miembro de la comunidad se debe regir por el manual de convivencia establecido e institucionalizado. la convivencia escolar está íntimamente ligada al proceso educativo donde los educandos aprenden a relacionarse con adultos, pares y más pequeños. Se coloca en escena el acercamiento con las ideas de los otros, a su vez a las diferencias, donde resulta vital el aprendizaje del respeto, la tolerancia y la inclusión. Otro de los propósitos fundamentales en la adquisición de un buen convivir es desarrollar la capacidad de transcender en los conflictos, desde la óptica de ser concebidos como oportunidades de crecimiento intelectual y personal.

Por medio de los procesos evaluativos de la institución se establece que un $45 \%$ de los estudiantes presentan una disminución académica debido al clima escolar, teniendo en cuenta que existen alteraciones disciplinarias dentro y fuera del salón de clases. El contacto con los acudientes ha permitido identificar que un porcentaje de padres, acudientes o cuidadores de los niños con mayor dificultad disciplinaria, asumen responsabilidad frente al actuar de los mismos, involucrándose activamente en lo concerniente a las estrategias propuestas en la presente investigación.

En la sede Adriano Puentes perteneciente a la IED Tercera Mixta; los estudiantes presentan comportamientos agresivos que deterioran la convivencia escolar, por lo cual el estudio busco fortalecer la convivencia escolar y solución de conflictos mediante la investigación como estrategia pedagógica, la cual permitirá generar estrategias de intervención para lograr una sana convivencia escolar, favoreciendo la creación de grupos de gestores de paz, los cuales serán conformados por estudiantes de cada curso que, además de orientar en temas de conflicto, violencia, resolución y mediación, estarán constituidos como semilleros de investigación que trabajen temas de paz.

\section{Convivencia escolar y solución de conflictos mediante la IEP}

Para López, Carvajal, Soto y Urrea (2013). convivir hace parte del reconocimiento de las personas con las que se debe compartir algún escenario o espacio de socialización, sin embargo, los sistemas sociales están sujetos a normas que favorecen la interacción con la finalidad de prevenir conflictos. Por su parte, la convivencia escolar hace mención al proceso de interrelación entre los miembros de un sistema educativo, estos presentan un conjunto de normas 
de comportamiento que deben cumplir los actores de la comunidad dentro del establecimiento, consignadas en el manual de convivencia. Según Good y Brophy (2011), la convivencia escolar es un proceso por el cual el sujeto adquiere o desarrolla una nueva conciencia y conocimiento de su capacidad de aprendizaje, para una mayor adaptabilidad en el ámbito escolar que le proporciona nuevos significados.

Para Chaparro, Caso, Fierro y Díaz (2015) esta se puede ser de 3 tipos:

a. La convivencia inclusiva reconoce la dignidad de todas las personas partiendo en reconocer un gran camino en pertinencia en una buena comunicación y relación entre los miembros de la familia haciendo más clara y comprensiva, más profunda, estrecha y gratificante para las personas, bien educadas, especialmente para los hijos. Para la comunicación familiar con calidad, sus principales ejes son es la identidad y cuidado, la valoración de las diferencias y la pluralidad. Destaca la importancia del reconocimiento de logros, esfuerzos y capacidades, el cuidado a las necesidades de otros, el trabajo colaborativo, así como el sentido de pertenencia al grupo de clase y a la comunidad escolar.

b. En este apartado se destaca la importancia de la convivencia democrática, que se refiere a una participación de responsabilidad enfocada en la generación y seguimiento a los acuerdos que regulan la vida en común, el dialogo en la familia entenderlo como un ambiente que facilita el encuentro mediante la importancia de la construcción colectiva de reglamentos y normas con enfoque de principios éticos; así como las decisiones participativas para la acción colectiva, el diálogo reflexivo y el manejo formativo de conflictos. c. La convivencia pacífica, que se constituye, en gran medida, en función de las anteriores, hace hincapié a la capacidad de establecer interacciones humanas basadas en el dialogo, vehículo de comunicación, mediante, el respeto y la tolerancia, la prevención y atención de conductas de riesgo, el cuidado de los espacios y bienes colectivos, la reparación del daño y la reinserción comunitaria. (p. 22).

Para Ramírez (2018), una sociedad plural como la nuestra requiere del respeto a la singularidad, basadas en una gestión para la sociedad del conocimiento, y convivencia y de orientar la propia vida, la defensa y la promoción de los valores comunes ante el cambio que esta facilitando nuevos enfoques administrativos en todos los sentidos operativos en la búsqueda en generar un valor agregado mediante una propuesta educativa que promueva un enfoque sistémico humanizan té hacia lo estratégico - dinamizador, inclusión de la ciencia y tecnología, capacidad de internacionalización; convirtiéndose en factores claves de competitividad, posibilitando permanencia en el contexto organizacional en un entorno globalizado.

Woolfolk (2012), menciona que el concepto de convivencia se refiere al accionar de grupos sociales democráticos, las relaciones entre sus miembros y las normas que estos comparten. Estas normas surgen de una concertación entre partes, las cuales se diferencian de la normativa establecida por premios y castigos. Por el contrario, la convivencia es un proceso en permanente construcción en cada institución educativa que debe ser aceptado e internalizado por cada uno de sus miembros.

De igual manera, Torrego y Moreno (2003) abordaban temas referidos a la convivencia y la disciplina en los centros escolares con el fin de dinamizar experiencias 
de innovación democrática en los centros educativos, conseguir una red plural de Centros por la Innovación Democrática, promover experiencias colaborativas $\mathrm{y}$, a la vez, apoyar la investigación en las escuelas que experimenten estas nuevas formas de colaboración.

La convivencia busca soluciones aplicables desde el punto de vista de la formación, tanto del profesorado como del alumnado, considerando que mejorar la convivencia desde una perspectiva de centro implica ir más allá de la resolución de problemas concretos; esto se consigue con una metodología de proceso donde se evalúa de forma cíclica, se abarca a la comunidad educativa completa, $\mathrm{y}$ plantean las estrategias en función de las necesidades encontradas (Torrego y Moreno, 2003).

Las actitudes agresivas son en efecto una alteración hacia la sana convivencia, en las diferentes manifestaciones físicas, verbales y psicológicas (Rodrí- guez, Almonacid y Marín, 2013). Afectando la convivencia escolar que trata de construir un modo de relación entre las personas de una comunidad, sustentada en el respeto mutuo y en la solidaridad recíproca, expresada en la interrelación armoniosa y sin violencia entre los diferentes actores y estamentos de la Comunidad Educativa (Ministerio de Educación de Chile, 2015).

El Grupo Gestor de Paz, nace de la necesidad de mejorar en la Institución las relaciones interpersonales; permitiéndole a los niños y jóvenes diferentes formas del manejo de los conflictos que se generan en su entorno escolar y en el grupo de amigos en el cual se desarrolla. Se logra sustentar la importancia de esta iniciativa mediante la implementación de estos espacios en las escuelas que son los lugares donde mayormente se concentran los niños y niñas durante gran parte de su tiempo diario (Bernal y Sierra, 2006).

TABLA 1

\section{Modelo ecológico de la prevención de la violencia}

\begin{tabular}{|c|c|}
\hline Individual & $\begin{array}{l}\text { Cabe decir que, este modelo pretende identificar los factores biológicos y de historia } \\
\text { personal que influyen en el comportamiento de una persona: impulsividad, } \\
\text { bajo nivel educativo, abuso de sustancias psicotrópicas y antecedentes de } \\
\text { comportamiento agresivo o de haber sufrido maltrato. }\end{array}$ \\
\hline Relacional & $\begin{array}{l}\text { Este apartado indaga el modo en el que las relaciones sociales cercanas aumentan } \\
\text { el riesgo de convertir a una persona en víctima o responsables de actos violentos. }\end{array}$ \\
\hline Comunitario & $\begin{array}{l}\text { Este apartado se enfoca en examinar los contextos de la colectividad en los } \\
\text { que se inscriben las relaciones sociales, como la escuela, el lugar de trabajo y } \\
\text { el vecindario, y busca identificar las características de estos contornos que se } \\
\text { asocian con ser víctimas o agresores. }\end{array}$ \\
\hline Social & $\begin{array}{l}\text { En efecto, este último modelo hace referencia en los factores sociales más } \\
\text { generales que determinan la tasa de evidencia. Se incluyen a que factores que } \\
\text { crean un clima de aceptación de la violencia, los que reducen las inhibiciones } \\
\text { contra esta, y los que crean y mantienen las brechas entre distintos segmentos } \\
\text { de la sociedad. }\end{array}$ \\
\hline
\end{tabular}

Fuente: elaboración propia tomado de Tuvilla, 2016. 
De los anteriores planteamientos se deduce que, esta perspectiva o enfoque permite analizar una situación dada, reduciendo su grado de violencia y acrecentando factores de protección como el deseo de la paz buscada como clima necesario e indiscutible para vivir en una sociedad auténticamente humanas, mediante una percepción positiva de sí mismo a través del desarrollo de la autoestima en la adquisición de competencias sociales, como la capacidad de pedir ayuda y reaccionar con energía ante las diferentes escenarios en manifestaciones de violencia; alcanzando un nivel de concienciación adecuado respecto a las diferentes formas o expresiones de la violencia; y adquirir habilidades y aptitudes que favorezcan el apoyo o auxilio entre víctimas y agresores

Cerdas (2015) concibe la educación como una herramienta poderosa para la construcción de paz, de modo que promueva el reconocimiento, comprensión y respeto de los derechos humanos, de la diferencia, justicia y concertación, logrando la interiorización individual y colectiva de relaciones pacíficas y armoniosas entre los seres humanos. Así mismo, Fernández (2006) asegura que para comprender la naturaleza de la escuela es necesario partir de un análisis crítico de la estructura social, como también de los intereses y conflictos que la conforman. Entendiendo que históricamente, la escuela ha sido un lugar donde se han presentado conflictos, pero esta también se ha preocupado por buscar y construir alternativas de solución. Por ello, la educación debe ser analizada desde una perspectiva diferente, asumiendo que ésta puede contribuir con la formación de ciudadanos capaces de modificar las relaciones sociales existentes.

La construcción de paz en la escuela no se concibe para evitar que se sigan generando conflictos, sino para permitir el abordaje de sus raíces estructurales, interviniendo a partir de la democracia y la justicia, construyendo espacios equitativos de participación estudiantil, donde se asegure que todos los sujetos tengan los medios para participar en el desarrollo de su propia sociedad, en ese sentido la convivencia debe estar planteada hacia una concepción democrática, propiciando la construcción de relaciones interpersonales, institucionales y culturales justas que ofrezcan a todos los estudiantes un acceso equitativo a la educación de calidad (Carbajal, 2013).

Por otro lado, la investigación es un proceso sistemático, organizado y objetivo, cuyo propósito es responder a una pregunta o hipótesis, aportando al aumento del conocimiento sobre un tema desconocido o poco estudiado. Así mismo, la investigación es una actividad sistemática dirigida a obtener, mediante observación y experimentación, nuevo conocimiento necesario para ampliar información en diversos campos de la ciencia y la tecnología. (Hernández, Fernández y Baptista, 2006). Por otro lado, la investigación se puede definir también como la acción y el efecto de realizar actividades intelectuales y experimentales de modo sistemático con el propósito de aumentar los conocimientos sobre una determinada materia y teniendo como fin ampliar el conocimiento científico (Roblero,2006).

De esta manera se comprende que la investigación posibilita la construcción de conocimiento, convirtiéndose en un instrumento para comprender una realidad o plantear soluciones frente a una problemática. Sin embargo, si integra a la educación se constituye como una base de la enseñanza, permitiendo al docente desde una reflexión crítica de la práctica pedagógica, elaborar nuevas estrategias que dinamizan el currículo, a su vez estas estrategias pueden dirigir procesos reales de transformación, no solo de prácticas pedagógicas sino también prácticas sociales inmersas en la escuela (Salcedo, 2000). La 
investigación como estrategia pedagógica ejerce un impacto positivo al integrarla al aula de clases, brindándole a la comunidad educativa la posibilidad de construir nuevo conocimiento apropiando herramientas científicas, que respondan a una necesidad o problemática social, proyectando al estudiante hacia un aprendizaje significativo (Ausubel, 2002).

Así mismo, la Investigación como Estrategia Pedagógica, tiene como finalidad instaurar bases científicas a los estudiantes en el aula de clases, esta parte de una dinámica metodológica, que busca dar respuestas a los interrogantes de los niños y niñas de la Institución y que asigna a los docentes un nuevo rol en el aula, para que en el momento indicado, puedan ser ellos mismos quienes busquen comprender el mundo que los rodea e interpretar y describir sus problemas en un lenguaje sustentado en la ciencia. En la implementación de la IEP, se parte de la pregunta del sentido común de los niños, niñas y jóvenes para reelaborarla en la perspectiva de la educación popular, lo que quiere decir que, reconoce la existencia de saberes comunes y elaborados así en la negociación con los conocimientos disciplinares, y de las maneras como se correlacionan en un convenio cultural (Mariño, 2010).

A los efectos de este, en la actualidad, existen propuestas metodológicas de enfoques basados en investigación, entendidos como la forma de materializar lo que se hace y como se implementa donde surgen infinidad de propuestas metodológicas que se basan en un enfoque o toman varios para hacer de ellos una propuesta metodológica; es el caso de las pedagogías basadas o fundadas en investigación, las cuales se desarrollan en diferentes contextos y asumen variados caminos en coherencia con los paradigmas y corrientes en los cuales se inscriben. Dentro de esta teoría basada en investigación se encuentra la Enseñanza por descubrimiento y el Programa On- das, con su propuesta de la Investigación como Estrategia Pedagógica (IEP), como una perspectiva que se desarrolla en ese enfoque y toma elementos de la pedagogía critico-liberadora y del enfoque socio-cultural (Mejía y Manjarrés, 2010).

Resulta oportuno, pensar que la investigación desde las corrientes educativas criticas significa una lucha teórico - práctica por la manera como sus presupuestos sobre el conocimiento, la ciencia, su epistemología, la cultura, lo humano y los grupos sociales enmarcan una acción que durante cuatrocientos años ha sido señalada como objetiva, lo que quiere decir que, no solo ha construido una forma de ella, sino que también ha ayudado a generar formas de poder que en la sociedad han servido para el control y la gestación de desigualdades, y en estos tiempos, nuevas formas de acumulación y dominación (Ortega, 2009).

La educación a lo largo del tiempo ha presentado cambios significativos, que invitan al docente a diseñar e implementar nuevas estrategias pedagógicas para dirigir el proceso de enseñanza con sus estudiantes, una estrategia pedagógica es un conjunto de actividades que se realizan con un orden lógico y coherente en función del cumplimiento de objetivos del plan académico. Es decir, es una planificación que contiene métodos o acciones que permiten al estudiante alcanzar los logros propuestos, mejorar su aprendizaje y facilitar su crecimiento personal (Picardo, Balmore, y Escobar, 2004).

Cochran y Litle (2006) plantearon la existencia de conocimientos y reflexiones en la acción, lo que permite integrar en las actuaciones explícitas e implícitas lo cognitivo, lo emocional, la teoría y la práctica. Reconociendo que tanto quien enseña como quien aprende dentro de una comunidad trabajan para generar conocimiento local, prever su práctica y teorizar sobre ella, interpretando las conclusiones de otros, todo lo anterior es posible al integrar la investigación al aula. 


\section{Metodología}

Se realizó un estudio de tipo cualitativo, utilizando el modelo de investigación acción, desde un diseño descriptivo, exploratorio. La investigación cualitativa busca conocer e interpretar la realidad de los participantes a través de sus propias experiencias, entregando una información subjetiva del fenómeno de estudio (Hernández, Fernández, y Baptista, 2010). Por su parte el modelo de investigación acción plantea una producción de conocimiento basado en la reflexión de los participantes, teniendo en cuenta la participación activa de maestros en el proceso de enseñanza y acompañamiento tecnológico, guiando al estudiante en su proceso de investigación. Generando nuevo saber y conocimiento sobre una realidad determinada en un proceso de aprendizaje colaborativo.

Los estudios con un diseño descriptivo están encaminados a especificar las características de la población sujeto de estudio o los fenómenos que sean sometidos a análisis. Bajo un estudio de tipo exploratorio, debido a que no se han realizado estudios previos en la institución (Hernández, Fernández, y Baptista, 2010). La población sujeto de estudio estuvo constituida por 180 estudiantes de básica primaria con edades que oscilan entre los 5 y 15 años, pertenecientes a la IED Tercera Mixta sede Adriano Puentes del municipio de Fundación Magdalena.

Para la recogida de datos se utilizó la observación participante y el diario de campo, estas son técnicas de recolección cualitativas que implican que el docente tome un rol activo en el proceso de investigación, donde no solo debe estar como espectador del proceso de implementación, sino que también debe participar del mismo, registrando cada elemento observado con relación a la variable de estudio. En el diario de campo se registran y sistematizan los elementos obtenidos de la observación participante donde el contacto con las personas del contexto, modulan las reflexiones y conclusiones que se pueden extraer.

La metodología del proyecto de investigación se realizó a partir de los recorridos de las trayectorias de indagación, basados en la integración de la IEP al aula, articulado al plan de estudios, transversalizando así la educación ambiental a las diversas asignaturas, lo anterior con la finalidad de generar una trasformación social y educativa a través del aprendizaje en contexto, involucrando intereses, motivaciones y realidades de los estudiantes para generar conocimiento científico

\section{Resultados}

A partir de la IEP que se puede definir como una metodología pedagógica que se centra en la investigación, esta surge por medio de las construcciones pedagógicas críticas que parten de las realidades latinoamericanas y que están en sintonía con los enfoques que van surgiendo y a su vez dinamizan a nivel mundial las diferentes perspectivas (Mejía, 2011).

Los estudiantes fueron capacitados por el cuerpo docente en temas de Conflicto, Violencia, Bullying, Mediación, Valores, Ética, Democracia y Ciudadanía. Determinando las características del proceso de formación en gestores de paz y estableciendo un comité de sana convivencia. Con talleres de estudiantes a estudiantes sobre los conceptos antes capacitados y ejercicios de mediación dentro y fuera del aula. Cumpliendo de las trayectorias de indagación y ejecución de estrategias de comités gestores de paz.

Mancomunadamente, los estudiantes expusieron preguntas que surgían en el proceso y generaron nuevas puestas de investigación. A partir de allí se genera el despliegue de la investigación como estrategia pedagógica según explica la ruta metodológica. La cual ayuda a promover la investigación como horizonte en la construcción del saber. 


\section{Conclusiones}

El objetivo de este programa es el de promover espacios de apropiación social del conocimiento en el que los niños aprendan a investigar, hagan preguntas y gestionen conocimiento para responder a preguntas de investigación que estén relacionadas con la vida cotidiana y el contexto donde se desenvuelven" (Colciencias, 2015).

Se reconoce la necesidad de implementar un plan de acción que garantice el sostenimiento de la sana convivencia, el bienestar de los miembros de la comunidad educativa y salvaguarde la integridad de los educandos de la sede Adriano Puentes. Motivo por el cual la propuesta se direcciona en el diseño de estrategias que converjan la investigación como estrategia pedagógica en la formación y establecimiento de grupos gestores de paz.

Generar el andar investigativo en los grupos gestores de paz busca alimentar conceptos, criterios, perspectivas a partir de nuevas formas pedagógicas, donde los estudiantes son activos en la adquisición del saber y paralelamente realizan el ejercicio de promover la transformación de su comunidad educativa a través de la instrucción/estrategias que brinden a sus compañeros y cuerpo docente. Se visualiza al grupo como arquitectos de estrategias que, desde su lugar de niños, provoquen mayor receptividad en la colectividad infantojuvenil.

A través de espacios de apropiación social a nivel institucional se presenta la propuesta de investigación, en conjunto con las sedes anexas a la Institución Educativa Departamental Tercera Mixta de Fundación, Magdalena. Se dispone del cuerpo docente, administrativos, asociación de padres de familia y representantes de estudiantes para la socialización de las estrategias.

Se vislumbra la participación de espacios de apropiación social del programa Ciclón. Además de llegar a las comunida- des aledañas a la sede, conforme a la gestión del grupo gestores de paz, teniendo en cuenta las acciones sociales que emprenda con las familias del sector.

\section{Referencias}

Ausubel, D. (2002). Adquisición y retención del conocimiento. Madrid: Paidós.

Bernal, C. y Sierra, L. (2006). Escuela territorio de paz. [Tesis]. Universidad de la Sabana, Chía. Recuperado de https:// www.google.com.co/url?sa=t\&rct=$\mathrm{j} \& \mathrm{q}=\&$ esrc $=\mathrm{s} \&$ source $=$ web\& $\mathrm{cd}=3 \& \mathrm{cad}$ $=$ rja\&uact $=8 \& v e d=0$ ahUKEwjPkdznw tXXAhUD22MKHf-rAKQQFggzMAI\& url=https $\% 3 \mathrm{~A} \% 2 \mathrm{~F} \% 2$ Fintellectum.unisabana.edu.co $\% 2$ Fbitstream $\% 2$ Fhandl e\%2F10818\%2F2064\%2F121728.pdf\% 3Fsequence\%3D1\%26isAllowed\%3Dy\& usg=AOvVaw3P6O8PXsy-JVFAPbvy4cIh

Carbajal, P. (2013). Convivencia democrática en las escuelas. Apuntes para una reconceptualizacion. Revista Iberoamericana de Evaluación Educativa, 6(2), 13-35.

Cerdas, E. (2015). Desafíos de la educación para la paz hacia la construcción de una cultura de paz. Recuperado de Redalyc: http://www.redalyc.org/articulo. oa?id=194138017009>

Chaparro, A., Caso, J., Fierro, M. y Díaz, C. (2015). Desarrollo de un instrumento de evaluación basado en indicadores de convivencia escolar democrática, inclusiva y pacífica. Perfiles Educativos, 20(149). 20-41.

Cochran, M. y Litle, S. (2003). Más allá de la certidumbre adoptar una actitud indagadora sobre la práctica. Barcelona: Octaedro.

Fernández, O. (2006). Una aproximación a la cultura de paz en la escuela. Revista Educere, 10(33). 
Good \& Brophy. (2011). Clima organizacional. Madrid: La Muralla.

López C, Carvajal C., Soto M. y Urrea, P. (2013). Factores asociados a la convivencia escolar en adolescentes. Rev. Educación y Educadores, 16(3). 383-410.

Hernández, R., Fernández, C. y Baptista, P. (2010). Metodología de la investigación. México, D.F.: Editorial Mc Graw Hill.

Manjarrés, M. y Mejía, M. (2011). Lineamientos del programa Ondas. Recuperado de http://repositorio.colciencias. gov.co:8081/jspui/handle/11146/361

Mariño, G. (2010). El diálogo en la educación de jóvenes y adultos. Dos propuestas pedagógicas para implementarlo. En, El taller dialógico/la recuperación de experiencias laborales. Bogotá; D.C.: OEI.

Mejía, M. y Manjarrés, M. (2010). La Investigación como Estrategia Pedagógica. En, La reconstrucción colectiva del Programa Ondas 2006-2008. (129-163). Bogotá, D.C.: Colciencias-Ondas.

Ortega, P., Peñuela, D. y López, D. (2009). Sujetos y prácticas de la pedagogía crítica. Bogotá, D.C.: Ediciones El Búho.

Picardo, O., Balmore, R. y Escobar, J. (2004). Diccionario enciclopédico de ciencias de la educación. San Salvador: El Salvador.

Ramírez, R. (2018). Tendencias emergentes de la gestión de talento humano en las organizaciones. En, M. Quintero y F. Sánchez, Responsabilidad Corporativa: una mirada integral en América Latina. (101-107). Programa Editorial Universidad del Valle, Cali.

República de Chile. Ministerio de Educación. (2015). Convivencia escolar. Recuperadodehttp://www.convivenciaescolar. cl/index2.php?id_seccion $=4010 \& i d$ _ portal $=50 \& i d \_$contenido $=17916$
República de Colombia. Departamento Administrativo de Ciencia, Tecnología e Innovación -Colciencias (2015). Los mejores maestros Ondas, los grandes maestros del país. Recuperado de http://www.colciencias.gov.co/sala_de_prensa/los-mejores-maestros-ondas-los-grandes-maestros-del-pais

República de Colombia. Congreso de la República. (2013). Por la cual se crea el sistema nacional de convivencia escolar y formación para el ejercicio de los derechos humanos, la educación para la sexualidad y la prevención y mitigación de la violencia escolar. [Ley 1620]. Diario Oficial No. 48.733. Recuperado de http://wsp. presidencia.gov.co/Normativa/Leyes/Documents/2013/LEY\%201620\%20 DEL\%2 015\%20DE\%20MARZO\%20 DE\%202013.pdf

República de Colombia. Ministerio de Educación Nacional. (2013). Por el cual se reglamenta la Ley 1620 de 2013, que crea el Sistema Nacional de Convivencia Escolar y formación para el ejercicio de los Derechos Humanos, la Educación para la Sexualidad y la Prevención y Mitigación de la Violencia Escolar. [Decreto 1965]. Diario Oficial No. 48.910. Recuperado de https://www.mineducacion.gov.co/1759/ articles-327397_archivo_pdf_proyecto_ decreto.pdf

Roblero, C. (2006). Técnicas y Procesos de la Investigación Científica. Guatemala.

Rodríguez, E., Almonacid, H. y Marín, J. (2013). Juegos tradicionales como estrategia metodológica para disminuir las actitudes agresivas. [Trabajo de grado]. Universidad Libre, Barranquilla.

Salcedo, R. (2000). Experiencias docentes, calidad y cambio escolar: investigación e innovación en el aula la investigación en el aula: y la innovación. Biblioteca virtual Luis Ángel Arango. Recuperado de http:// www.banrepcultural.org/blaavirtual/ educacion/expedocen/expedocen8a.htm. 
Torrego, J. y Moreno, J. (2003). Convivencia y Disciplina en la escuela. El aprendizaje de la Democracia. Madrid: ALIANZA.

Tuvilla, J. (2016). Convivencia Escolar, y Resolución pacífica de conflictos. Andalucía: Junta de Andalucía.
Valenzuela, G. y Rochin, L. (2013). Libertad de expresión y su impacto en la democracia. Jurídicas CUC, 9(1), 89100.

Woolfolk, A. (2012). Diseño de la Organización. Bogotá, D.C.: Legis. 